\title{
Principais patologias laríngeas em professores
}

\author{
Main laryngeal pathologies in teachers
}

\section{Principales patologías laríngeas en profesores}

\author{
Sther Soares Lopes da Silva*
}

\begin{abstract}
Resumo
Introdução: Os docentes estão expostos a diversos fatores de riscos ocupacionais, e acredita-se que qualidade vocal não está relacionada apenas com ausência de alteração, mas sim com bem-estar geral do indivíduo. Existem vários fatores que contribuem para o aparecimento de problemas vocais nos professores como: o uso inadequado da voz, competição vocal, sobrecarga vocal, hábitos inadequados como exemplo (cigarro e álcool). Objetivo: O presente estudo teve como objetivo analisar as principais alterações vocais em professores e consistiu em uma pesquisa bibliográfica, descritiva de caráter qualitativo. Método: Realizou-se um levantamento da literatura nacional e internacional, publicada nos idiomas Inglês, Espanhol, Português entre 2007 e 2017. Foram selecionados por meio das bases de dados "Biblioteca Virtual em Saúde" (BVS) e "Scientific Electronic Library" (SciELO), utilizando-se os descritores, professores, patologias da voz, saúde vocal e prevenção. Resultados: Observou-se que as principais patologias encontradas foram os nódulos, pólipos, edema de Reinke, cisto e sulco vocal. A rouquidão é o principal sintoma descrito pelos docentes, mas é preciso se preocupar com os demais sintomas sendo eles: fadiga vocal, falhas na voz, falta de ar, ardência na garganta, esforço para falar. Conclusão: Verificou-se na literatura estudada a sugestão de adotar uma política pública de saúde com o objetivo de educar e conscientizar os docentes quanto a noções de higiene vocal, estratégias de comunicação, economia vocal e valorização da profissão, que são ferramentas necessárias para diminuir os índices elevados de docentes com algum tipo de alteração vocal.
\end{abstract}

Palavras-chave: Docentes; Voz; Doenças da laringe; Saúde do trabalhador; Prevenção de doenças.

\section{Abstract}

Introduction: Teachers are exposed to several occupational risk factors, and it is believed that vocal quality is related not only to absence of change, but to general well-being of the individual. There are

* Faculdade Unyleya, Brasília, DF, Brasil

Contribuição do autor:

SSLS: Percepção do estudo, Coleta de dados, Organização dos dados coletados, Elaboração do artigo, Metodologia, Resultado e Discussão

E-mail para correspondência: Sther Soares Lopes Da Silva stherrs@hotmail.com

Recebido: 15/03/2018

Aprovado: 17/09/2018 
several factors that contribute to the development of vocal problems in teachers such as: inadequate use of voice, vocal competition, vocal overload, inappropriate habits as an example (cigarette and alcohol). Objective: This study aimed to analyze the main vocal changes in teachers and consisted of a descriptive bibliographical research of a qualitative nature. Method: A survey of the national and international literature was published in English, Spanish and Portuguese between 2007 and 2017. The descriptors, teachers, voice pathologies, and vocal health were selected using the "Biblioteca Virtual em Saúde" (BVS) and "Scientific Electronic Library" (SciELO) databases. Results: It was observed that the main pathologies found were nodules, polyps, Reinke's edema, cyst and vocal groove. Hoarseness is the main symptom described by the teachers, but it is necessary to worry about the other symptoms being: vocal fatigue, voice failures, shortness of breath, burning throat, effort to speak. Conclusion: It was verified in the studied literature the suggestion of adopting a public health policy with the objective of educating and making teachers aware of notions of vocal hygiene, communication strategies, vocal economy and valorization of the profession are necessary tools to reduce the indices of teachers with some type of vocal alteration.

Keywords: Faculty; Voice; Laryngeal Diseases; Occupational Health; Disease Prevention.

\section{Resumen}

Introducción: Los docentes están expuestos a diversos factores de riesgo ocupacional, y se cree que calidad vocal no está relacionada sólo con ausencia de alteración, sino con el bienestar general del individuo. Hay varios factores que contribuyen a la aparición de problemas vocales en los profesores como: el uso inadecuado de la voz, competencia vocal, sobrecarga vocal, hábitos inadecuados como ejemplo (cigarrillo y alcohol). Objetivo: El presente estudio tuvo como objetivo analizar las principales alteraciones vocales en profesores y consistió en una investigación bibliográfica, descriptiva de carácter cualitativo. Métodos: Se realizó un estudio de la literatura nacional e internacional, publicado en los idiomas Inglés, español, portugués entre 2007 y 2017 fueron seleccionados a través de bases de datos "Biblioteca Virtual em Saúde" (BVS) y "Scientific Electronic Library" (SciELO), utilizando los descriptores, los maestros, las enfermedades de la voz, salud vocal y prevención. Resultados: Se observó que las principales patologías encontradas fueron los nódulos, pólipos, edema de Reinke, quiste y surco vocal. La ronquera es el principal síntoma descrito por los docentes, pero hay que preocuparse por los demás síntomas que son: fatiga vocal, fallas en la voz, falta de aire, ardor en la garganta, esfuerzo para hablar. Conclusión: Se verificó en la literatura estudiada la sugerencia de adoptar una política pública de salud con el objetivo de educar y concientizar a los docentes en cuanto a nociones de higiene vocal, estrategias de comunicación, economía vocal y valorización de la profesión son herramientas necesarias para disminuir los índices altos de docentes con algún tipo de alteración vocal.

Palabras claves: Docentes; Voz; Enfermedades de la Laringe; Salud Laboral; Prevención de Enfermedades.

\section{Introdução}

Os profissionais da voz, dentre eles os professores, são todos os que utilizam a voz como instrumento de trabalho. Os docentes estão expostos a diversos fatores de riscos ocupacionais, e acredita-se que qualidade vocal não está relacionada apenas com ausência de alteração, mas sim com bem-estar geral do indivíduo. É importante que o profissional conheça bem o instrumento de trabalho (estruturas que compõe a produção vocal) a fim de prevenir futuras alterações laríngeas e adotar medidas de prevenção.

No campo da docência, a voz é um dos principais meios de comunicação entre professor e aluno e os professores constituem um grupo de risco a desenvolver problemas vocais ${ }^{1}$. A voz do professor está vulnerável a influências negativas ocorridas do mau uso vocal e fonotraumas, sendo estes fatores comumente associados ao desconforto do trato vocal e a queixas vocais ${ }^{2}$. 
Os distúrbios da voz fazem parte do contexto do professor, sendo a voz o instrumento de trabalho. As alterações vocais tornam-se um problema para o docente, podendo diminuir ou impedir de lecionar. É preciso se atentar para doenças laríngeas mais comuns nos professores e correlacionar com o nexo causal, principalmente para o CID 10 (J - 38.0 e R - 49.0) $)^{3}$.

Os fatores sócio-ambientais que fazem parte da rotina do professor também são considerados como um fator de risco para a saúde vocal e podem evoluir para uma disfonia ${ }^{4}$.

Existem vários fatores que contribuem para 0 aparecimento de problemas vocais nos professores, como: o uso inadequado da voz, competição vocal, sobrecarga vocal, hábitos inadequados como exemplo (cigarro e álcool). Por isso, é preciso se atentar aos principais sinais e sintomas das alterações vocais, sendo: rouquidão persistente, tosse seca, dor na região da garganta, pigarro, a voz geralmente é mais grave, falhas na voz, boca seca, diminuição no volume da voz gerando um esforço para falar, ardência, gritar, sensação de corpo estranho na garganta 5 .

As principais causas das alterações vocais são: a sobrecarga vocal, pois a maioria apresenta mais de um cargo, as salas de aula geralmente com muitos alunos e a maioria das escolas não apresenta recursos para a economia vocal, principalmente escolas públicas de periferia e zona rural. Observa-se um número elevado de professores com pouca noção de higiene e economia vocal, o que leva ao desgaste precoce da musculatura laríngea a problemas de voz e posterior ao absenteísmo. Esta falta ao trabalho acaba gerando um custo para as escolas, governo e atraso no cronograma de ensino aos alunos ${ }^{6}$.
Por isso, é importante uma política pública de saúde com o objetivo de diminuir o adoecimento dos profissionais da voz o que gera o absenteísmo. Observa-se que a maioria dos beneficiários do INSS em auxílio-doença por problemas vocais são profissionais da voz, sendo a categoria mais requerente a dos professores ${ }^{7}$.

Diante do exposto, este estudo teve como objetivo verificar quais as principais patologias laríngeas que acometem os professores por meio de revisão bibliográfica qualitativa e descritiva.

\section{Método}

O presente estudo trata-se de uma revisão bibliográfica que busca descrever as principais patologias vocais encontradas nos professores. Para isso, realizou-se um levantamento da literatura nacional e internacional, publicada nos idiomas Inglês, Espanhol, Português entre 2007 e 2017. A amostra foi selecionada por meio das bases de dados, "Biblioteca Virtual em Saúde" (BVS) e "Scientific Electronic Library" (SciELO), utilizando-se os descritores: professores, voz profissional, patologias da voz, saúde vocal e prevenção.

Foram investigados na literatura, em periódicos nacionais e internacionais de fonoaudiologia, artigos para a elaboração deste estudo.

A análise inicial dos artigos foi feita através do titulo e resumo. Aqueles artigos que não estavam em concordância com o objetivo proposto do estudo foram eliminados, totalizando em uma amostra final de 14 artigos.

Os artigos foram distribuídos em um (quadro 1) para melhor visualização e, posteriormente foram descritos os principais achados de cada artigo. 
Quadro 1. Distribuição dos artigos identificados na literatura

\begin{tabular}{|c|c|c|}
\hline Ano & Autor & Título \\
\hline 2008 & $\begin{array}{l}\text { Silverio, Gonçalves' Penteado, } \\
\text { Vieira, Libard, Rossi29. }\end{array}$ & $\begin{array}{l}\text { Ações em saúde vocal: proposta de melhoria do perfil vocal de } \\
\text { professores. }\end{array}$ \\
\hline 2009 & $\begin{array}{l}\text { Luchesi, Mourão, Kitamura, } \\
\text { Nakamura }{ }^{25} \text {. }\end{array}$ & $\begin{array}{l}\text { Problemas Vocais no Trabalho: prevenção na prática docente } \\
\text { sob a óptica do professor. }\end{array}$ \\
\hline 2009 & $\begin{array}{l}\text { Azevedo, Vianello, Oliveira, Oliveira, } \\
\text { Oliveira, Silva21. }\end{array}$ & $\begin{array}{l}\text { Queixas vocais e grau de disfonia em professoras do ensino } \\
\text { fundamental. }\end{array}$ \\
\hline 2010 & Cardim, Behlau, Zambon ${ }^{18}$ & $\begin{array}{l}\text { Sintomas vocais e perfil de professores em um programa de } \\
\text { saúde vocal. }\end{array}$ \\
\hline 2011 & $\begin{array}{l}\text { Musial, Dassie-Leite, Zaboroski, } \\
\text { Casagrande } 22 .\end{array}$ & $\begin{array}{l}\text { Interferência dos sintomas vocais na atuação profissional de } \\
\text { professores }\end{array}$ \\
\hline 2011 & Caporossi, Ferreira ${ }^{19}$. & $\begin{array}{l}\text { Sintomas vocais e fatores relativos ao estilo de vida em } \\
\text { professores. }\end{array}$ \\
\hline 2012 & $\begin{array}{l}\text { Lima-Silva, Ferreira, Oliveira, Silva, } \\
\text { Ghirardi12. }\end{array}$ & $\begin{array}{l}\text { Distúrbio de voz em professores: autorreferência, avaliação } \\
\text { perceptiva da voz e das pregas vocais. }\end{array}$ \\
\hline 2012 & $\begin{array}{l}\text { Santos, Almeida, Paula, Ribeiro, } \\
\text { Oliveira }^{27} \text {. }\end{array}$ & Comunicador eficaz: a voz do professor e saúde preventiva. \\
\hline 2013 & $\begin{array}{l}\text { Pizolato, Mialhe, Cortellazzi, } \\
\text { Ambrosano, Cornacchionirehder, } \\
\text { Pereira }^{4} \text {. }\end{array}$ & $\begin{array}{l}\text { Avaliação dos fatores de risco para distúrbios de voz em } \\
\text { professores e análise acústica vocal como instrumento de } \\
\text { avaliação epidemiológica. }\end{array}$ \\
\hline 2013 & Masson, Loiola, Fabron, Horigüela ${ }^{1}$. & $\begin{array}{l}\text { Aquecimento e Desaquecimento Vocal em Estudantes de } \\
\text { Pedagogia. }\end{array}$ \\
\hline 2014 & Valente, Botelho, Silva?. & $\begin{array}{l}\text { Perfil dos beneficiários do INSS em auxílio-doença por } \\
\text { distúrbios benignos da voz. }\end{array}$ \\
\hline 2015 & Cielo, Ribeiro, Hoffmann ${ }^{5}$. & Sintomas vocais de futuros profissionais da voz. \\
\hline 2016 & $\begin{array}{l}\text { Ferreira, Giannini, Alves, Brito, } \\
\text { Andrade, Latorre } 6 .\end{array}$ & Distúrbio de voz e trabalho docente. \\
\hline 2016 & Silva, Almeida, Lucena, Silva ${ }^{17}$. & Sintomas vocais e causas autorreferidas em professores. \\
\hline
\end{tabular}

\section{Resultado e discussão}

As principais alterações vocais encontradas nos professores são: os nódulos, pólipos, edema de Reinke, cisto, sulco vocal. O professor apresenta risco elevado para desenvolver distúrbio de voz de origem ocupacional, devido à exposição a diversos fatores relacionados ao ambiente de trabalho.

Os nódulos vocais são lesões benignas da laringe devido ao mau uso da voz associados a hábitos inadequados. São tipicamente bilaterais e ocorre na porção membranácea média das pregas vocais verdadeiras, lugar onde ocorre maior força de contato durante a vocalização ${ }^{8}$.

As principais características das disfonias são a rouquidão, seguida da fadiga vocal, dor na garganta e falhas na voz ao longo do dia. O diagnóstico é feito pelo médico otorrinolaringologista através da videolaringoscopia ou videolaringoestroboscopia. Depois de diagnosticada a lesão iniciará a terapia fonoaudiológica, através do repouso vocal, noções de higiene vocal e exercícios para reabsorver a lesão?.
Os pólipos são lesões de massa benigna localizados no interior da lâmina própria na porção membranosa média das pregas vocais. Porém, os pólipos podem ser encontrados na porção contralateral e também podem envolver praticamente toda a extensão da prega vocal, sendo estes predominantemente unilaterais. A ocorrência é devido a um período de abuso vocal, embora possa ocorrer depois de um incidente traumático como um grito intenso $^{8}$.

Os cistos aparecem nas margens das pregas vocais e às vezes localizam-se na superfície superior, são predominantemente unilaterais e com frequência confundidos com nódulos e pólipos. $\mathrm{O}$ edema de Reinke é uma reação natural do tecido a algum trauma ou uso inadequado ou excessivo vocal, ou pode ser concomitante a uma infecção ou inflamação. Além do abuso vocal o edema de Reinke crônico é mais comumente associado ao fumo, sendo que ocorre com maior frequência em mulheres, principalmente se fumantes há muito tempo ${ }^{8}$. O edema de Reinke provoca uma diminuição do "pitch" vocal, devido ao aumento de massa das pregas vocais ${ }^{10}$. 
Sulcos vocais são lesões benignas da laringe nas quais é observado um entalhe ao longo da linha média superior da prega vocal. A causa ainda é incerta, podendo ser de etiologia a mau uso e abuso vocal. O uso inadequado da voz sugere comportamentos de produção vocal que se distorcem da propensão normal do mecanismo fonatório de trabalhar efetiva e eficientemente. A produção suave da voz pode ser alterada de diversas maneiras, sendo que cada um de nós pode produzir voz de variadas formas, algumas mais eficientes, outras não ${ }^{8}$.

As alterações vocais causam grande impacto no contexto social, psicológico e profissional do docente, interferindo na qualidade de vida, sendo este um fator importante, pois interfere no bem estar físico e psicológico do professor. A disfonia é qualquer alteração que impede a emissão natural da voz, e está diretamente relacionada com a qualidade de vida do indivíduo ${ }^{11}$.

A falta de conscientização, conhecimento e estrutura física do ambiente de trabalho contribuem para o índice elevado de professes com problemas de voz. O professor é o profissional mais investigado em relação aos distúrbios de voz, devido a inúmeras alterações encontradas na classe, característica esta ligada ao contexto profissional ${ }^{12}$.

É preciso conscientização para prevenir as alterações vocais, pois os impactos negativos na saúde do docente são inúmeros, tanto no contexto profissional como psicológico e social. Vários são os fatores para os problemas vocais; os distúrbios de voz em professores são consequência de fatores externos, sendo que as condições ambientais e organizacionais a que o docente é exposto e a falta de conhecimento de técnicas vocais contribuem para $\mathrm{o}$ adoecimento destes profissionais ${ }^{13}$.

Nesse caso, há uma tendência para que o professor não considere a disfonia como risco ocupacional, julgando que os problemas de voz sejam inerentes à docência, não considerando a necessidade de prevenir ou tratar o problema, que passa a ser vista como uma consequência natural da profissão. O professor precisa se conscientizar de que seus problemas de voz podem ser relacionados ao seu trabalho. ${ }^{13}$

Em contrapartida, esta incapacidade de perceber as causas e alterações coloca em perigo o profissional da voz ao expor que alguns professores, por falta de conhecimento, desconhecem a própria voz, o que dificulta a percepção de alteração na qualidade vocal, assim como não associam os sintomas vocais ao uso incorreto da voz. Em virtude desses comprometimentos com a saúde vocal, muitos professores preferem continuar lecionando mesmo apresentando vários sintomas e não se preocupam com a qualidade da voz. É provável que os professores se preocupem mais com a funcionalidade da voz e não com a qualidade vocal ${ }^{13-14}$.

Competir com o ruído dos alunos, ventiladores, ou ruído externo é outro fator agravante para o professor. Deve-se evitar este hábito e adotar alguma estratégia para economizar a voz. Ao competir com esses tipos de ruído gera uma sobrecarga da musculatura laríngea e atrito nas pregas vocais; tanto o ruído ambiental externo como no interior da sala de aula, fazem com que os professores elevem a intensidade vocal acarretando uma sobrecarga na musculatura laríngea, e, além disso, podem gerar estresse no docente. O ruído e a acústica ruim são fatores negativos da sala de aula, além da violência na escola e da falta de recursos físicos, que contribuem para o desgaste vocal do professor. No ambiente de trabalho, vários fatores contribuem para o aparecimento de traumas nas pregas vocais, como a sobrecarga vocal, estresse do trabalho, tempo da profissão, poeira no local de trabalho, trabalho repetitivo ${ }^{15-16}$.

Sabe-se que o envelhecimento do corpo humano traz uma série de mudanças às vezes desconfortáveis, sendo que a voz também sofre esse impacto. Falta de projeção, falta de ar, cansaço vocal são alguns sinais de envelhecimento da voz. Quanto mais aumenta a idade, maior a chance de desenvolver um problema vocal. Dessa forma, verifica-se que muitos professores brasileiros enfrentam fatores externos juntamente com predisposição de fatores biológicos do próprio indivíduo, o que leva ao afastamento da sala de aula ${ }^{17}$.

$\mathrm{O}$ estresse associado ao esgotamento físico pode ser considerado como outra causa de alteração vocal. A insatisfação com o trabalho gera um desequilíbrio emocional e muitos são os fatores podendo incluir neste item a automedicação, o uso em excesso de álcool e principalmente quando associado ao cigarro e uso de drogas ilícitas. Os autores associam o desenvolvimento do estresse levando à síndrome de Burnout devido à sobrecarga de trabalho e instabilidade na profissão, afetando então, o emocional do professor. Muitos docentes relatam que escrever em quadro de giz, ficar em pé por muito tempo, carregar o material didático, levar trabalho para casa e a falta de um local para repouso 
aumenta o estresse e promove o desequilíbrio emocional. A automedicação é um dos problemas entre os docentes, pois muitos não procuram o médico o que acentua o risco de piorar a saúde ${ }^{16}$.

Outro fator que compromete a voz do professor é o uso excessivo de álcool e tabagismo com a associação do uso contínuo da voz e a presença de hábitos inadequados. É importante que os professores introduzam na vida diária hábitos saudáveis com a voz e procurem sempre um profissional habilitado a fim de orientá-los sobre higiene vocal, diminuindo assim, o risco das alterações vocais ${ }^{18}$.

Por isso, é importante verificar as causas, sintomas das alterações vocais e listar quais medidas de controle e prevenção. É relevante conhecer as causas/sintomas das alterações vocais para adotar uma política de prevenção de promoção à saúde do trabalhador (professor), como triagem vocal de professores em escolas, com o objetivo de elaborar ações que visem à prevenção de alterações vocais promovendo assim, qualidade de vida no trabalho $(\mathrm{QVT})^{17}$.

Sintoma consiste na manifestação de modificações orgânica ou funcional, sendo então uma queixa em que o paciente pode relatar sensações associadas à fonação, como uma dor na região do pescoço ou a sensação de irritação na garganta ${ }^{8}$. O sintoma vocal é uma queixa em que o indivíduo relata o que sente de diversos modos, abordando sensações relacionadas à fonação, como dor no pescoço ou de garganta após conversações prolongadas ${ }^{19}$.

A rouquidão é um dos sintomas mais apresentados pelos professores e, além disso, pode estar presente a otalgia, disfagia, odinofagia, tosse seca e pigarro constante. Pacientes com história de rouquidão, superior a três semanas de duração, devem ser examinados, por meio de laringoscopia ${ }^{20-21}$.

É fundamental perceber qualquer alteração na qualidade vocal e verificar a presença dos sintomas. Em muitos casos, observa-se o afastamento do professor do seu local de trabalho devido a complicações nas pregas vocais, tendo como sintomas a rouquidão seguida de voz fraca, falta de ar, falhas na voz, cansaço vocal, ausência da voz, dor local, pigarro, tosse, garganta seca, principalmente quando os professores estão em atividades ${ }^{17-18}$.

É importante ressaltar que os professores apresentam mais de um sintoma, e é nítida a presença de dificuldades na comunicação, nas relações pessoais e com os demais profissionais da escola ${ }^{22}$.
Os hábitos inadequados com a voz são fatores para desencadear os sintomas, associados à falta de conscientização. Os professores usam a voz de maneira intensa e muitas vezes sem noção de cuidados e higiene vocal, cometendo assim, abusos vocais constantemente ${ }^{19}$.

Os achados quanto à sintomatologia vocal destaca que a rouquidão é a queixa mais referida pelos docentes e nota-se uma dificuldade dos mesmos em perceber os primeiros sintomas de alterações na voz, traduzindo assim, desconhecimento sobre a voz e falta de autopercepção, sendo este um fator determinante ${ }^{23}$.

É importante ressaltar que, se os sintomas se intensificam podem evoluir para uma doença laríngea, e as consequências dos sintomas vocais acarretam prejuízo ao professor tanto na vida profissional, como social e psicológica. Por este motivo é importante ter consciência sobre as alterações vocais ${ }^{22}$.

A disfonia relacionada ao trabalho também pode estar associada a sintomas de sofrimento mental devido às exigências do ambiente de trabalho e, geralmente, os pacientes associam o início do sintoma a algum fato estressante em suas vidas. A pressão que existe em responder a essas exigências, o medo do desemprego, a falta de informação e outras contingências do mundo faz com que o trabalhador suporte esses sintomas e continue trabalhando, até que haja um agravamento do quadro clínico, exigindo intervenção terapêutica ${ }^{24}$.

A voz é o instrumento de trabalho do docente e apesar das novas tecnologias nada substitui a voz do professor. O índice de professores com problemas vocais aumentou significativamente nos últimos anos, e novas pesquisas estão sendo realizadas com o objetivo de prevenir as alterações vocais. Ainda, é importante aprimorar a qualidade vocal do professor para prevenir as alterações. No entanto, é preciso que os docentes percebam a importância em identificar os sintomas dos problemas vocais, com o intuito de prevenir e procurar o mais rápido possível à assistência médica ${ }^{25}$.

Um fator preocupante trata-se do ruído que é elevado no ambiente escolar e dever ser tratado. A NBR-10.152 da ABNT, preconiza que o nível de ruído em ambiente escolar esteja entre 40 a 50 $\mathrm{dB}(\mathrm{A})$. Entretanto, é comum encontrar ruído acima do nível estabelecido, o que prejudica o docente tanto na voz como na saúde física, pois o excesso de ruído pode acarretar tontura, náuseas, insônia, 
problemas circulatórios, digestivos, irritabilidade além de interferir no processo de comunicação ${ }^{25}$. Se o nível de ruído fosse menor os educadores posteriormente não utilizariam a voz com tanta intensidade, o que foi observado no estudo dos autores, em que foi realizada a relação entre os valores médios do nível de pressão sonora das vozes das professoras e do ruído ambiental (NPS), com e sem crianças e constatou-se correlação positiva entre intensidade vocal e ruído ambiental durante as aulas com a presença das crianças ${ }^{15}$.

Os professores apresentam um conhecimento limitado em relação à voz e por isso, é fundamental programas de saúde vocal para esta classe de profissionais. Os programas de saúde vocal contribuem satisfatoriamente para o comportamento vocal do professor sendo possível observar a diminuição dos sintomas, intensidade da voz controlada, diminuição do desgaste vocal, melhor projeção vocal, redução da tensão cervical entre outros.

Os programas de saúde vocal são importantes, pois mostram ao professor como cuidar da voz, sendo de fundamental importância na grade curricular dos acadêmicos, pois quando começarem a lecionar terão maior percepção dos problemas vocais e provavelmente maior cuidados para evitar problemas futuros ${ }^{11}$.

Um exemplo de ações é o Programa de saúde vocal do professor (PSVP) criado pelo Estado de Minas Gerais que promove educação e conscientização aos professores.

Sobre o programa de saúde vocal, a equipe de fonoaudiologia realizou palestras presenciais em escolas estaduais do interior e até março de 2012, foram capacitados no PSVP 119.444 professores. Em relação ao tratamento, os professores que apresentam distúrbios vocais foram encaminhados ao Instituto de Previdência do Servidor Público (IPSEMG), para agendamento do início do tratamento fonoaudiológico ${ }^{27}$.

É importante que o professor realize o aquecimento e desaquecimento vocal para manter a qualidade vocal e evitar problemas vocais. Ainda, é fundamental aquecimento e desaquecimento para a musculatura durante o desempenho das atividades ${ }^{28}$.

Além do aquecimento e desaquecimento vocal é fundamental manter hábitos saudáveis $\mathrm{como}^{29}$ :

1. Evitar fumar; pois o cigarro agride todo trato respiratório, bem como as pregas vocais; e o uso de drogas ilícitas como (crack, cocaína, maconha, entre outras), pois a agressão é ainda maior no trato respiratório e organismo no geral;

2. Evitar pigarrear, pois mesmo sendo um movimento involuntário acaba acarretando um atrito nas pregas vocais;

3. É importante prestar atenção em relação à tosse, pois as pregas vocais ficam batendo uma com a outra;

4. Evitar gritar, pois o grito agride ainda mais as pregas vocais;

5. Evitar o uso do ar condicionado, pois a exposição provoca ressecamento de todo trato vocal;

6. Evitar falar em ambiente ruidoso;

7. O uso de sprays e pastilhas só mascara os sintomas, agravando ainda mais o quadro. É fundamental procurar o médico otorrinolaringologista para uma avaliação.

8. Evitar roupas apertadas na região do diagrama e acessórios que dificultam a produção da fala como brincos, colares.

9. A mudança de temperatura é outro fator, pois provoca o choque térmico, causando edemas na musculatura da laringe.

10. Devem-se tratar as alergias que são um fator de alterações para as pregas vocais.

11. É fundamental fazer sempre repouso vocal, uma boa noite de sono ajuda a manter a qualidade vocal.

12. Alimentação saudável: é fundamental evitar alimentos pesados que dificultem a respiração;

13. Ingerir de 2 a 3 litros de água natural ao longo do dia.

14. Evitar leite e derivados, o que aumenta a secreção de todo trato vocal.

Sabe-se que a disfonia é um grande fator de ausência no trabalho, por esse motivo vem sendo estudada por vários pesquisadores e aponta urgência de novas pesquisas com medidas preventivas, relacionado ao ambiente de trabalho do professor ${ }^{30}$. Quando se fala em prevenção acredita-se que é preciso dar "voz" ao professor, conhecer melhor o ambiente de trabalho, recursos físicos da escola entre outros, ou seja, verificar a real necessidade do docente e assim, construir ações eficazes ${ }^{25}$.

Além disso, é interessante a presença de fonoaudiólogos nas escolas para as questões relacionadas à voz e psicólogos para as questões de saúde mental, lembrando que esta assistência não é de caráter assistencialista e sim preventivo. As ações multidisciplinares devem complementar a grade 
curricular do discente, a fim de aprender noções de economia e saúde vocal, colocando assim, em prática as ações estudadas durante a graduação ${ }^{25}$.

As alterações vocais em professores precisam ser estudadas e deve-se abordar o quanto antes as formas de prevenção, sendo importante que a legislação brasileira adote ações preventivas, com o objetivo de diminuir as alterações vocais e identificar precocemente os sintomas e o diagnóstico ${ }^{2}$.

Os programas de saúde vocal são importantes, pois visam à melhoria das alterações vocais nos educadores, considerando que a disfonia é um sintoma que interfere não só no processo de comunicação, mas na saúde física, psicológica e social do individuo e muitas vezes o professor, ou por falta de conhecimento ou por que ainda consegue dar aulas, mesmo apresentando o sintoma, não procura um profissional especializado o que leva ao agravamento e piora do quadro de disfonia ${ }^{31}$.

\section{Conclusão}

A voz é o instrumento de trabalho do professor, e considerando que as alterações vocais interferem nas relações interpessoais, é fundamental manter uma boa qualidade vocal.

Foi possível verificar as principais patologias laríngeas que acometem os professores, dentre eles os nódulos, pólipos, edema de Reinke, cisto, sulco vocal e os principais sintomas que desencadeiam tais patologias. A rouquidão ainda é o principal sintoma que afeta a qualidade vocal do professor, mas além de identificar a rouquidão é preciso se preocupar com os demais sintomas, sendo eles: fadiga vocal, sensação de corpo estranho na garganta, falhas na voz, falta de ar, ardência na garganta, esforço para falar, perda da potência da voz. Verificou-se que o professor demonstra resistência em procurar atendimento especializado, quando apresenta algum tipo de sintoma vocal. $\mathrm{O}$ ambiente de trabalho nem sempre é o ideal, falta estrutura física as salas de aulas são lotadas, o professor geralmente trabalha em mais de uma escola, o que acaba ocasionando uma fadiga na musculatura da laringe. A falta de educação dos discentes contribui para o aumento do estresse nos professores levando a problemas físicos e emocionais, o que acabará interferindo na qualidade vocal. Alem disso, a alimentação não é adequada, ( o café, a água gelada, alimentos densos como o chocolate, leite e derivados) ocasiona pigarro, excesso de muco, gerando assim, esforço ao falar.

Então, pode-se concluir que é preciso adotar medidas de política pública de saúde com o objetivo de educar e conscientizar os docentes quanto a noções de higiene vocal, estratégias de comunicação, economia vocal e valorização da profissão. É através de uma política pública e investimentos voltados para a saúde vocal do professor que se conseguirá diminuir as patologias vocais e reduzir o número de professores ajustados devido a problemas vocais. Como sugestão de pesquisas é fundamental investir em programas que aborde noções de higiene vocal, estratégias de economia vocal, técnicas facilitadoras de comunicação e triagem periódica dos professores. É através da conscientização e prevenção que será possível diminuir esses índices elevados de educadores com algum tipo de alteração vocal.

\section{Referências Bibliográficas}

1. MASSON MLV,LOIOLACM, FABRONE M, HORIGÜELA MLM. Aquecimento e Desaquecimento Vocal em Estudantes de Pedagogia. Distúrb Comum. 2013; 25(2): 177-185

2. SOUZA LBR, PERNAMBUCO LA, LIMA CR, SANTOS MM. Desconforto no trato vocal em professores do ensino fundamental. Rev Ciênc. Méd. Biol, 2015; 14(1): 36-41, jan./abr.

3. FERRACCIU CCS, ALMEIDA M. Distúrbio de voz relacionado ao trabalho do professor e a legislação atual. Rev. CEFAC. 2014; Mar-Abr; 16(2):628-633.

4. PIZOLATO RA, MIALHE FL, CORTELLAZZI KL, AMBROSANO GMB, CORNACCHIONIREHDER MIB. PEREIRAAC. Avaliação dos fatores de risco para distúrbios de voz em professores e análise acústica vocal como instrumento de avaliação epidemiológica. Rev CEFAC. 2013; Jul-Ago; 15(4): 957-966.

5. CIELO CA, RIBEIRO VV, HOFFMANN CF. Sintomas vocais de futuros profissionais da voz. Rev. CEFAC. 2015; Jan-Fev; 17(1): 34-43.

6. FERREIRA LP, GIANNINI SPP, ALVES NLL, BRITO AF, ANDRADE BMR, LATORRE MRD. Distúrbio de voz e trabalho docente. Rev CEFAC. 2016; Jul-Ago; 18(4):932-940.

7. VALENTE AMSL, BOTELHO C, SILVA AMC apud ARAÚJO RP. Perfil dos beneficiários do INSS em auxíliodoença por distúrbios benignos da voz. Rev Bras Med Trab. 2014; 12(1): 1-7.

8. COLTON RH, CASPER JK, LEONARD R. Compreendendo os problemas da voz. Rio de Janeiro: Revinter; 2010.

9. BASSI RDS. Identificação inteligente de patologias do trato vocal. [Trabalho de conclusão de curso]. São Carlos (SP): Universidade de São Paulo. Escola de engenharia de São Carlos; 2013. 
10. MACHADO JM. Características acústicas de patologias vocais no português europeu, 2015. In: VERDOLINI K, ROSEN CA, BRANSKY RC. Classification Manual for Voice Disorders I. Rev Soc Bras Fonoaudiol. 2007; 12(1): 72-5.

11. RICARTE A, OLIVEIRA G, BEHLAU M. Validação do protocolo Perfil de Participação e Atividades Vocais no Brasil. CoDAS 2013; 25(3): 242-9.

12. LIMA-SILVA, MFB, FERREIRA LP, OLIVEIRA IB, SILVA MAA, GHIRARDI ACAM. Distúrbio de voz em professores: autorreferência, avaliação perceptiva da voz e das pregas vocais. Rev Soc Bras Fonoaudiol. 2012; 17(4): 391-7.

13. PROVENZANO LCFA, SAMPAIO TMM. Prevalência de disfonia em professores do ensino público estadual afastados de sala de aula. Rev. CEFAC.2010; Jan-Fev; 12(1): 97-108.

14. FABRÍCIO MZ, KASAMA ST, MARTINEZ EZ. Qualidade de vida relacionada à voz de professores universitários. Rev. CEFAC. 2010; Mar-Abr; 12 (2): p.280-7.

15. GUIDINI RF, BERTONCELLO F, ZANCHETTA S, DRAGONE MLS. Correlações entre ruído ambiental em sala de aula e voz do professor. Rev Soc Bras Fonoaudiol. 2012; 17(4): 398-404.

16. SERVILHA EAM, ARBACH MP. Queixas de saúde em professores universitários e sua relação com fatores de risco presentes na organização do trabalho. Distúrb Comum. 2011; 23(2): 181-191.

17. SILVA GJ, ALMEIDAAA, LUCENA BTL, SILVA, MFB L. Sintomas vocais e causas autorreferidas em professores. Rev. CEFAC. 2016; Jan-Fev; 18(1): 158-166.

18. CARDIM KC, BEHLAU M, ZAMBON F. Sintomas vocais e perfil de professores em um programa de saúde vocal. Rev. CEFAC. 2010; Set-Out; 12(5): 811-819.

19. CAPOROSSI C, FERREIRA LP. Sintomas vocais e fatores relativos ao estilo de vida em professores. Rev. CEFAC. 2011; Jan-Fev; 13(1):132-139.

20. BRASIL. Ministério da Saúde. Secretaria de Vigilância em Saúde. Departamento de Saúde Ambiental e Saúde do Trabalhador. Protocolo de distúrbio de voz relacionado ao trabalho. Brasília, 2011.
21. AZEVEDO LL, VIANELLO L, OLIVEIRA HGP, OLIVEIRA IA, OLIVEIRA BFV, SILVA CM. Queixas vocais e grau de disfonia em professoras do ensino fundamental. Rev Soc Bras Fonoaudiol. 2009; 14(2): 192-6.

22. MUSIAL PL, DASSIE-LEITE AP, ZABOROSKI AP, CASAGRANDE RC. Interferência dos sintomas vocais na atuação profissional de professores. Distúrb Comum. 2011; 23(3): 335-341

23. MORAIS EPG, AZEVEDO RR, CHIARI BM. Correlação entre voz, autoavaliação vocal e qualidade de vida em voz de professoras. Rev. CEFAC. 2012; Set-Out; 14(5): 892-900.

24. Hermes EGC, BASTOS PRH. Prevalência de sintomas vocais em professores na rede municipal de ensino em Campo Grande-MS. Rev. CEFAC. 2015; Set-Out; 17(5): 1541-1555.

25. LUCHESI KF, MOURÃO LF, KITAMURA S, NAKAMURA, HY. Problemas Vocais no Trabalho: prevenção na prática docente sob a óptica do professor. Saúde Soc. 2009; 18(4): 673-681.

26. SEPLAG. Superintendência central de Perícia médica e saúde ocupacional. [atualizada em 2016 outubro; acesso em 2017 dezembro 13]. Disponível em: www.portaldoservidor. mg.gov.br/acesso-a-informacao/acoes-programas-e-outros/ programa-saudevocal-do-professor//.

27. SANTOS AS, ALMEIDA DM, PAULA LG, RIBEIRO MÀ, OLIVEIRA MP. Comunicador eficaz: a voz do professor e saúde preventiva. Revista de Eletrônica de Educação da Faculdade Araguaia. 2012; 2(2): 551-563.

28. PINHO SMR. Manual de higiene vocal para profissionais da voz. $4^{\mathrm{a}}$ ed. Pró-Fono. São Paulo; 2007.

29. SILVERIO KCA, GONÇALVES CGO, PENTEADO RZ, VIEIRA TPG, LIBARD A, ROSSI D. Ações em saúde vocal: proposta de melhoria do perfil vocal de professores. Pró-Fono Revista de Atualização Científica. 2008 jul-set;20(3).

30. MESTRE LR, FERREIRA LP. O impacto da disfonia em professores: queixas vocais, procura por tratamento, comportamento, conhecimento sobre cuidados com a voz, e absenteísmo. Rev Soc Bras Fonoaudiol. 2011; 16(2): 240-1. 